\title{
Renal atrophy after stereotactic body radiotherapy for renal cell carcinoma
}

\author{
Takaya Yamamoto ${ }^{\text {* }}$, Noriyuki Kadoya ${ }^{1}$, Ken Takeda ${ }^{1}$, Haruo Matsushita', Rei Umezawa' ${ }^{1}$, Kiyokazu Sato², \\ Masaki Kubozono', Kengo Ito', Yojiro Ishikawa', Maiko Kozumi', Noriyoshi Takahashi', Yu Katagiri', \\ Hiroshi Onishi ${ }^{3}$ and Keiichi Jingu ${ }^{1}$
}

\begin{abstract}
Background: Renal atrophy is observed in an irradiated kidney. The aim of this study was to determine dose-volume histogram parameters and other factors that predict renal atrophy after 10-fraction stereotactic body radiotherapy (SBRT) for primary renal cell carcinoma (RCC).

Methods: A total of 14 patients (11 males, 3 females) who received SBRT for RCC at Tohoku University Hospital between April 2010 and February 2014 were analyzed. The median serum creatinine level was $1.1 \mathrm{mg} / \mathrm{dl}$ and two patients had a single kidney. Nine patients were implanted with fiducial markers. The median tumor diameter was $30 \mathrm{~mm}$. SBRT was delivered at $70 \mathrm{~Gy}$ in 10 fractions for 7 tumors, at $60 \mathrm{~Gy}$ in 10 fractions for 2 tumors, and at $50 \mathrm{~Gy}$ in 10 fractions for 5 tumors with 6 and/or 15 MV X-ray using 5 to 8 multi-static beams. Renal atrophy was assessed using post-SBRT CT images after 12-24 months intervals. Correlations were examined by Spearman rank correlation analysis. Differences between two groups were evaluated by the Mann-Whitney test, and pairwise comparisons were made by the Wilcoxon signed-rank test.

Results: The median tumor volume shrunk from $14.8 \mathrm{cc}$ to $10.6 \mathrm{cc}(p=0.12)$, and the median irradiated kidney volume changed from $160.4 \mathrm{cc}$ to $137.1 \mathrm{cc}(p<.01)$. The median peak creatinine level was $1.6 \mathrm{mg} / \mathrm{dl}$ after treatment $(p<.01)$. Percentage volumes of the irradiated kidney receiving at least $10 \mathrm{~Gy}\left(V_{10}, p=0.03\right), V_{20}(p<.01), V_{30}$ $(p<.01), V_{40}(p=0.01)$, mean irradiated kidney dose $(p<.01)$, and magnitude of overlap between PTV and kidney volume $(p=0.03)$ were significantly correlated with post-treatment irradiated kidney volume in percent, and $V_{20}-V_{30}$ had strong correlation $(r<-0.70, p<.01)$. Patients with implanted fiducial markers showed a significantly lower ratio of renal atrophy $(p=0.02)$.
\end{abstract}

Conclusions: Significant renal atrophic change was observed. Dose distribution of SBRT at 20-30 Gy had a strong correlation with renal atrophy when irradiation was performed in 10 fractions.

Keywords: Stereotactic radiotherapy, SRT, Renal cell cancer, Renal atrophy, Renal remodeling

\section{Background}

Much progress has been made in extracranial stereotactic radiotherapy since the creation of a stereotactic body frame and application of the frame to treatment [1]. In thoracic malignancies, stereotactic body radiotherapy (SBRT) has become one of the most powerful local therapies and one of the most important treatment options for early stage non-small cell lung cancer, especially in

\footnotetext{
* Correspondence: t.yamamoto@rad.med.tohoku.ac.jp

'Department of Radiation Oncology, Graduate School of Medicine, University of Tohoku, Sendai, Japan

Full list of author information is available at the end of the article
}

elderly or inoperable patients [2]. Furthermore, pooled analysis of the results of a recent prospective trial have showed that survival rate and regional recurrence rate after SBRT for stage I non-small cell lung cancer were comparable to those after lobectomy [3]. Due to the progress in image-guided radiotherapy, SBRT has been applied to many other sites [4-6]. SBRT has sometimes been used as a definitive treatment for localized prostate cancer [4]. There has been an increasing number of reports on SBRT for hepatocellular carcinoma with a high rate of local control and acceptable toxicities [5]. 
For renal cell carcinoma (RCC), surgical resection (i.e., nephrectomy) has been the standard treatment. One of the reasons is that RCC is considered to be a radioresistant tumor, but the kidney itself is considered to be relatively radiosensitive [7]. Thus, radiotherapy for RCC has been performed as palliative radiotherapy in most cases or as postoperative radiotherapy in a limited setting $[8,9]$. However, in this SBRT era, several outcomes of SBRT for primary RCC have been reported. Although most of the clinical outcomes were reported from single institutions, local control rates were $84-100 \%$ and toxicities rates were relatively low [10-13]. In Japan, a clinical trial has been performed, and our institute took part in the trial starting in April 2010 [14]. This is the multicenter single arm clinical trial of SBRT for RCC, 50 Gy to 70 Gy in 10 fractions were prescribed within dose constraints of the organ at risk and primary endopoints were toxicity and 3-year local progression-free rate.

Because the kidney is a radiosensitive organ, renal atrophy developed after abdominal radiotherapy despite exposures to relatively low doses. A dose-volume histogram $(\mathrm{DVH})$ of renal atrophy 12 months after conventional radiotherapy has been reported [15]. They reported that percentage volumes of the primary irradiated kidney receiving at least 10 Gy, 15 Gy and 20 Gy were significantly associated with renal atrophy. This renal remodeling is also seen 12 months or more after SBRT, but, to the best of our knowledge, there has been only a single case report about post-SBRT renal atrophy [16]. Svedman et al. reported SBRT for 7 renal lesions in patients with one functional kidney [17]. Their results showed that SBRT was safe, and none of their patients required dialysis. Thus, SBRT for RCC is sometimes required for preserving the postoperative remaining kidney. Although SBRT focuses a high dose to a local region with a high level of accuracy, more studies are needed to achieve a higher level of safety. In the present study, DVHs of kidneys treated with a 10-session scheme of SBRT at our institute were retrospectively analyzed. DVH predictive factors for renal atrophy after SBRT intervals of 12-24 months and other factors affecting renal atrophy were investigated.

\section{Methods}

\section{Patients}

Before treatment, all patients with RCC whose maximum tumor diameter was $50 \mathrm{~mm}$ or less were assessed by a urologist. For patients who were judged to be inoperable, patients who were judged to be operable but for whom SBRT was preferred and patients who refused surgery, a radiation oncologist assessed the indication for SBRT. SBRT for RCC was performed for 17 consecutive patients at our institute between April 2010 and February 2014. Of those patients, eligibility criteria for current study were availability of follow-up CT images taken 12-24 months after SBRT, no requirement for hemodialysis before SBRT and no additional invasive therapy (such as nephrectomy or radiofrequency ablation) having been performed. Two patients underwent hemodialysis before SBRT and one operable patient declined to continue SBRT and underwent nephrectomy. A total of 14 patients with 14 tumors were retrospectively analyzed. The pretreatment characteristics of the patients are summarized in Table 1. Two patients had previously undergone nephrectomy and therefore had only one kidney. Nine patients were implanted with one to two gold fiducial markers in the kidney parenchyma near the tumor before SBRT. None of the patients had a histologically proven tumor, and assessment by radiologists and a urologist was therefore needed. First, the dynamic $\mathrm{CT}$ and MRI were interpreted by two or more radiologists, and agreement with radiographic diagnosis of RCC was needed. Second, the patients underwent examination by a urologist. Operability was judged by the urologist. Assessment that the patient was inoperable, the patient was operable but SBRT was better option than surgical resection, or the patient was operable but refused surgery was needed. Finally, patients received an explanation from a radiation oncologist, and written informed consent was obtained by the radiation oncologist. This study was approved by the Ethical Committee of Tohoku University

Table 1 Baseline characteristics of patients

\begin{tabular}{ll}
\hline Characteristics & No. (\%) \\
\hline Age, median, y/o & 75 (range: 58-87) \\
Fender & $3(21)$ \\
Male & $11(79)$ \\
Creatinine, median, mg/dl & 1.1 (range: 0.4-2.0) \\
Hypertension & $14(100)$ \\
Yes & $0(0)$ \\
No & \\
Diabetes mellitus & $7(50)$ \\
Yes & $7(50)$ \\
No & $10(71)$ \\
Administration of antithrombotic agents & $4(29)$ \\
Yes & \\
No & $2(14)$ \\
Past history of nephrectomy & $12(86)$ \\
Yes & \\
No & $9(64)$ \\
Fiducial markers & $5(36)$ \\
Yes & 30 mm (range: 16-46 mm) \\
No &
\end{tabular}


Hospital (2011-100), and informed consent was obtained from all patients.

\section{Treatment protocol and SBRT procedure}

Each patient was immobilized in the supine position with a body frame (Vac-loc, Med-tek, Orange City, IA), and movement of the implanted fiducial marker or diaphragm was measured to estimate respiratory tumor motion using continuous X-ray images in a simulator (Ximatron or Acuity system, Varian Medical Systems, Palo Alto, CA). To control respiratory movement, abdominal compression was used in 6 patients and the breath hold technique was used in 1 patient. All patients underwent a planning $\mathrm{CT}$ scan at a slice thickness of $2 \mathrm{~mm}$ with a multi-detector CT scanner (GE Light Speed Qxi, GE Healthcare, Waukesha, WI); 7 patients underwent a fast CT scan, 5 patients underwent a 4-dimensional CT scan and 2 patients underwent a slow-rotation $\mathrm{CT}$ scan (4 s/slice). The internal margin was determined from a planning CT image and from movement of the implanted fiducial marker or diaphragm.

Gross tumor volume (GTV) was defined as visible extent of the tumor on planning CT images, sometimes using CT and MRI fusion images. Clinical target volume (CTV) was equal to GTV. Internal target volume (ITV) was expansion of CTV for the internal margin. A planning target volume (PTV) margin of $5 \mathrm{~mm}$ around the ITV was added for patient positioning and set-up uncertainty.

The SBRT plan was created with a 3-dimensional radiotherapy planning system (Eclipse, Varian Medical Systems, Palo Alto, CA), and an analytical anisotropic algorithm (AAA version 8.6.15) was used for dose calculation. Fifty Gy in 10 fractions, 60 Gy in 10 fractions or 70 Gy in 10 fractions covering $95 \%$ of the PTV (D95) was delivered. The prescribed dose was selected on the basis of the highest dose within dose constraints of the organ at risk (Table 2). Seven tumors were prescribed 70 Gy in 10 fractions, 2 tumors were prescribed 60 Gy

Table 2 Dose constraints for planning organ at risk volume of 10-fraction SBRT

\begin{tabular}{lll}
\hline Organ & Constraints & Volume \\
\hline $\begin{array}{l}\text { Irradiated kidney (patient with } \\
\text { bilateral kidneys) }\end{array}$ & $30 \mathrm{~Gy}$ & Mean \\
$\begin{array}{l}\text { Irradiated kidney (patient with } \\
\text { single kidney) }\end{array}$ & $26 \mathrm{~Gy}$ & Mean \\
Lung & $20 \mathrm{~Gy}$ & $\leq 20 \%$ of total volume \\
Spinal cord & $35 \mathrm{~Gy}$ & Any point \\
Stomach, intestine & $52 \mathrm{~Gy}$ & $\leq 10 \mathrm{cc}$ \\
& $43 \mathrm{~Gy}$ & $\leq 100 \mathrm{cc}$ \\
Other organs & $71 \mathrm{~Gy}$ & $\leq 1 \mathrm{cc}$ \\
& $58 \mathrm{~Gy}$ & $\leq 10 \mathrm{cc}$ \\
\hline
\end{tabular}

in 10 fractions and 5 tumors were prescribed 50 Gy in 10 fractions. In only one case, 50 Gy in 10 fractions was delivered to the isocenter to meet dose constraints. The median isocenter dose was 68.8 Gy (range: 50.0-76.4 Gy). SBRT was delivered with a linear accelerator (Clinac 23EX, Varian Medical Systems, Palo Alto, CA) using 6 MV and/or $15 \mathrm{MV}$ X-ray beams with 5 to 8 coplanar and non-coplanar multi-static ports. SBRT was performed on consecutive treatment days. Concomitant tyrosine kinase inhibitor or interferon was not administered.

\section{Follow-up after SBRT}

Patients underwent follow-up examinations every 3 months for 3 years by a radiation oncologist and urologist. CT and MRI were also performed every 6 months for 3 years. When CT examination was performed, enhanced CT images were recommended but were not essential because it was expected that serum creatinine had worsened. Patients often underwent ultrasound examinations of the abdomen, but there were no protocol requirements about ultrasound.

\section{Renal atrophy assessment}

Each functional kidney parenchyma was defined as the contoured kidney avoiding renal cysts and renal pelvis (Fig. 1). Kidney volume was defined as functional kidney parenchyma minus GTV. Pretreatment functional kidney parenchyma was delineated using planning CT images. When the slow-rotation scanning technique was used for radiotherapy planning, the use of pretreatment diagnostic $\mathrm{CT}$ and planning $\mathrm{CT}$ rigid fusion images were permitted to avoid overestimation of kidney volume. When the noncontrast enhanced CT images were used for radiotherapy planning, the use of diagnostic MRI and planning CT rigid fusion images were permitted because there was no difference between CT and MRI for volumetry [18]. Post-SBRT kidney volume was assessed using follow-up diagnostic CT images with a slice thickness $\leq 2 \mathrm{~mm}$, and the use of follow-up CT and follow-up MRI fusion images was permitted as appropriate. Volumetric analysis was performed using Eclipse. The DVH parameter regarding kidney volume was analyzed using $V_{n}(\%)$, which was defined as percentage volume of the kidney receiving at least $n$ dose in Gy. Percent changes in parameters was defined as postSBRT parameters divided by pre-SBRT parameters in percent.

\section{Statistical analysis}

Time to an event was calculated from the first day of SBRT to the day an event was confirmed. Toxicity was graded according to the National Cancer Institute Common Terminology Criteria for Adverse Events version 4.0. Correlations between continuous variables were examined by Spearman's rank correlation, and $r$ was the correlation coefficient. Simple linear regression was applied to create 


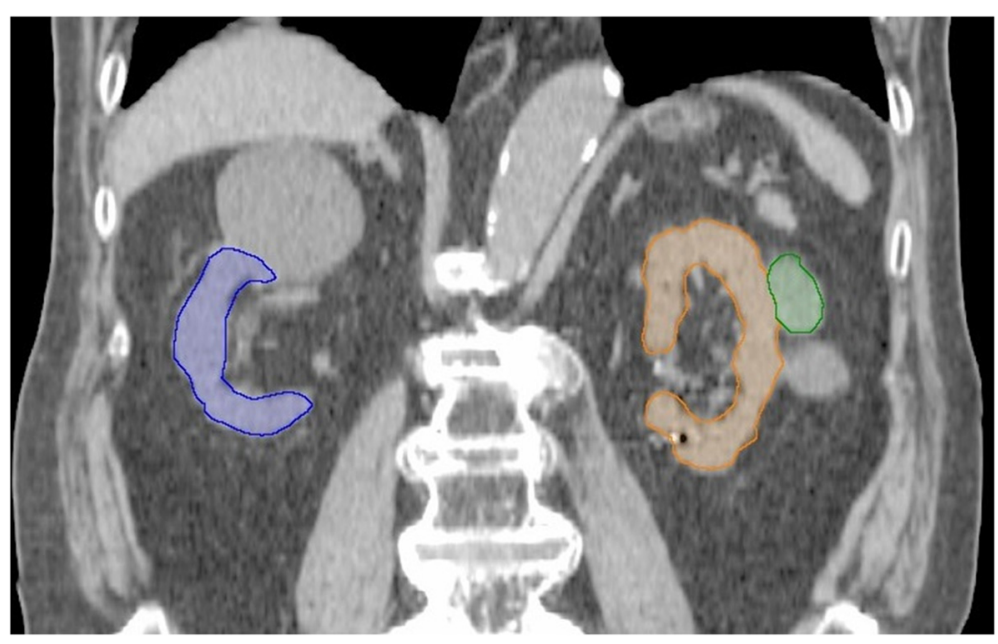

Fig. 1 Delineation of functional kidney parenchyma avoiding renal cysts and renal pelvis

a linear regression equation. Differences in continuous variables between two groups were evaluated by the Mann-Whitney test. Changes from pre-SBRT parameters to post-SBRT parameters were assessed using the Wilcoxon signed-rank test. A $p$-value $<.05$ was defined as significant in all tests. JMP Pro v.11.2 (SAS Institute, Cary, NA) was used for statistical analyses.

\section{Results}

\section{Toxicity and parameter changes}

The median interval between SBRT and renal atrophy assessment CT was 16.9 months (range: 12.0-21.8 months), and no patient was administered tyrosine kinase inhibitor or interferon during that interval. The median tumor volume shrunk from $14.8 \mathrm{cc}$ (range: 3.0-55.6 cc) to 10.6 cc (range: $1.3-38.9 \mathrm{cc}, p=0.12$ ). The median posttreatment tumor volume in percent was $73.4 \%$ (range: 41.5-144.6\%). Change in median irradiated kidney volume was from 160.4 cc (range: 99.4-295.5 cc) to $137.1 \mathrm{cc}$ (range: 70.6-258.7 cc, $p<.01$ ). The median post-treatment irradiated kidney volume in percent was $82.6 \%$ (range: 61.3-96.4 \%, Fig. 2). Change in median contralateral kidney volume was from $147.0 \mathrm{cc}$ (range: $118.1-183.6 \mathrm{cc}$ ) to 143.9 cc (range: $114.5-191.8 \mathrm{cc}, p=0.73$ ). The median post-treatment contralateral kidney volume in percent was $99.3 \%$ (range: 89.4-109.0 \%, Fig. 2). The median follow-up period for all patients was 31.2 months (range: 16.2-54.2 months). During follow-up, no grade 2 or higher renal and gastrointestinal toxicity occurred and there was no intervention. None of the patients had progression of hypertension and none of the patients required hemodialysis. The pre-SBRT median serum creatinine level was $1.1 \mathrm{mg} / \mathrm{dl}$ (range: $0.4-2.0 \mathrm{mg} / \mathrm{dl}$ ). The post-SBRT peak value seen in intervals of 0.3-48.1 months was
$1.6 \mathrm{mg} / \mathrm{dl}(p<.01)$, and the post-SBRT value at the time of reporting was $1.3 \mathrm{mg} / \mathrm{dl}(p=0.05)$. Serum creatinine of each patient over time was showed in Fig. 3.

\section{Univariate analysis}

Spearman's correlations between post-treatment irradiated kidney volume in percent and continuous variables are shown in Table 3, and the results of MannWhitney test for differences between post-treatment irradiated kidney volume in percent and categorical variables are shown in Table 4. Significant correlation were seen for $\mathrm{V}_{10}(r=-0.56, p=0.03), \mathrm{V}_{15}(r=-0.68$, $p<.01), \mathrm{V}_{20}(r=-0.76, p<.01), \mathrm{V}_{30}(r=-0.71, p<.01)$, $\mathrm{V}_{40}(r=-0.65, p<.01)$, mean irradiated kidney dose $(r=-0.66, p<.01)$, and magnitude of overlap between PTV and kidney volume $(r=-0.56, p=0.03)$, on the other hand, PTV did not have a correlation $(r=-0.03, p=0.91)$. The observed factor of post-treatment contralateral kidney volume in percent also had correlation $(r=0.65, p=0.02)$. There was a significant difference between the posttreatment irradiated kidney volume in percent with and without fiducial markers. In patients with fiducial markers and those without fiducial markers, the post-treatment irradiated kidney volumes in percent were $87.5 \%$ (range: 75.6-96.4\%) and 71.4\% (range: $61.3-83.4 \%, p=0.02$ ), respectively, but the median isocenter doses were $73.4 \mathrm{~Gy}$ (range: 50.0-76.4 Gy) and 65.5 Gy (range: 53.7-73.6 Gy), respectively, and the isocenter doses were not significantly different $(p=0.54)$. Further detailed investigation using Spearman's correlations revealed that $V_{24}$ showed the strongest correlation $(r=-0.778, p<.01)$. A simple linear regression for the post-treatment irradiated kidney volume in percent and $V_{24}$ was used for predicting a linear equation. As a result, the following linear regression equation was obtained: 


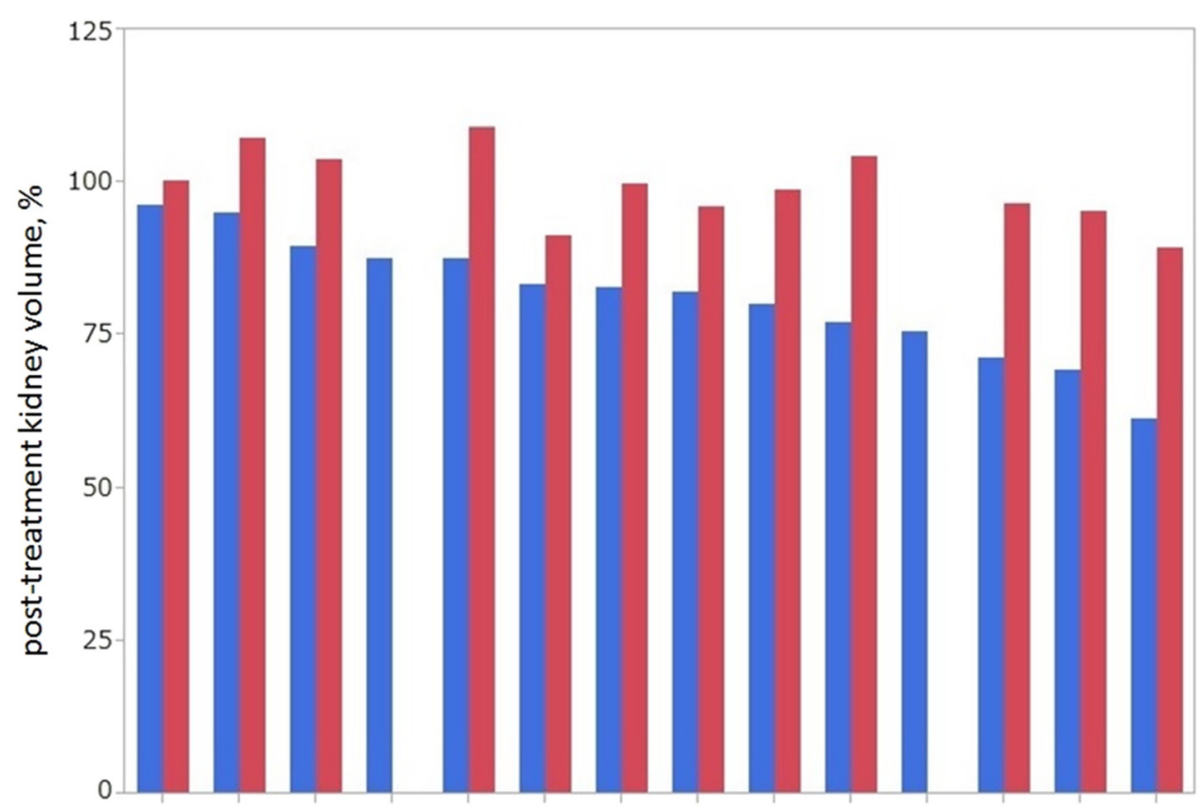

Patients

Fig. 2 Each median percent change in irradiated kidney volume (blue) and contralateral kidney volume (red)

Estimated post-treatment irradiated kidney volume(\%) $=111.4-0.659 * \mathrm{~V}_{24}(\%)+\varepsilon$

Epsilon is the error term and its standard deviation is 6.43. The coefficient of determination was $0.60(p<.01$, Fig. 4).
Analysis for the contralateral kidney was also performed $(n=12)$. The DVH for the contralateral kidney showed that median $\mathrm{V}_{5}$ was $0.0 \%$ (range: $0.0-16.0 \%$ ), $\mathrm{V}_{10}$ was $0.0 \%$ (range: $0.0-0.4 \%$ ), and median value of mean contralateral kidney dose was 1.1 Gy. Post-treatment contralateral kidney volume in percent was $99.3 \%$ (range: 89.4-109.0 \%).

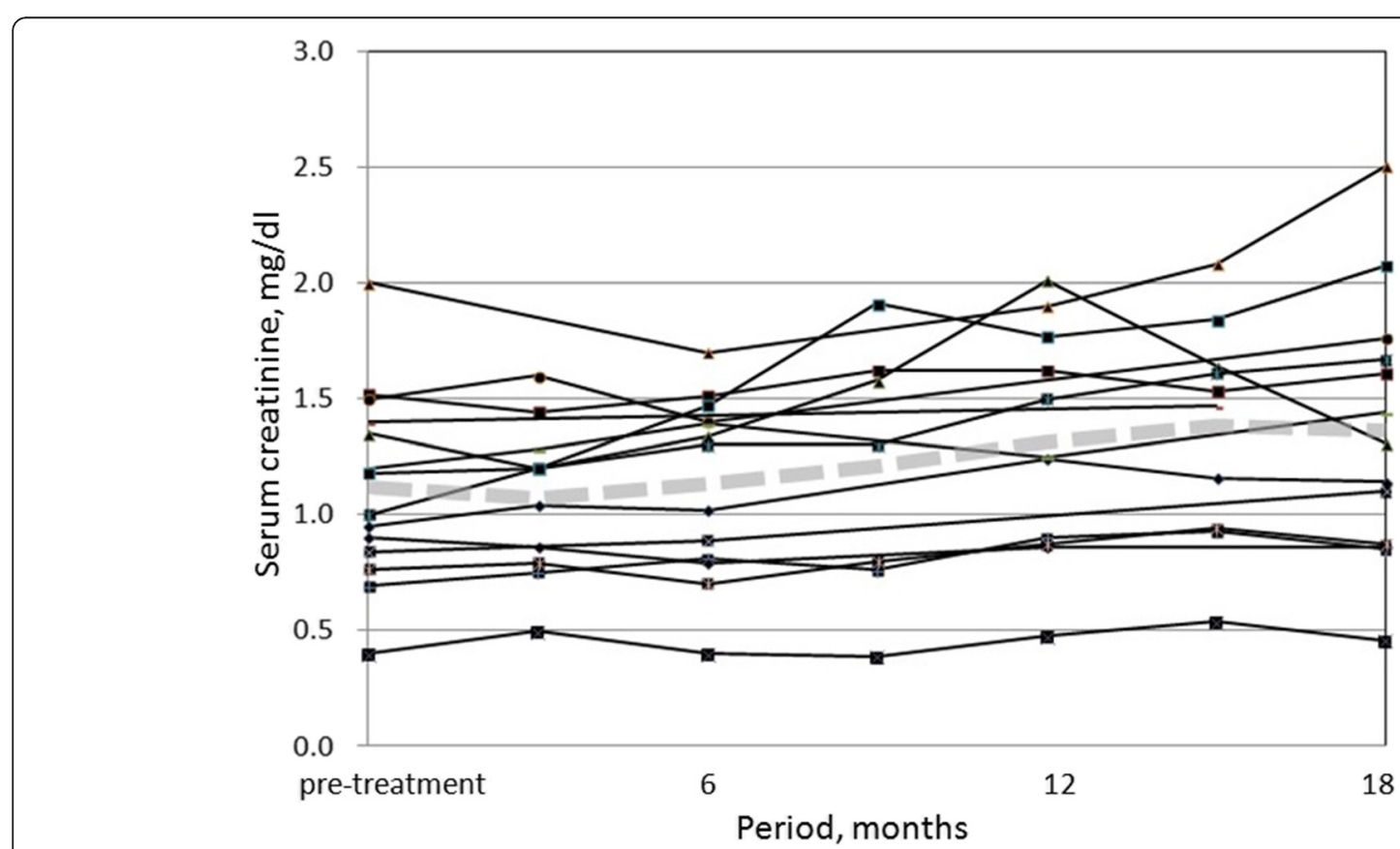

Fig. 3 Serum creatinine levels over time. Figure 3 shows changes in serum creatinine levels of each patient over time, and the grey dashed line shows mean serum creatinine over time 
Table 3 Spearman's correlations between post-treatment irradiated kidney volume in percent and continuous variables

\begin{tabular}{|c|c|c|c|c|}
\hline \multirow[b]{2}{*}{ Variables } & \multicolumn{2}{|c|}{ Parameters } & \multicolumn{2}{|c|}{ Correlation of irradiated kidney } \\
\hline & Median & Range & Correlation coefficient & $P$ value \\
\hline$V_{5}(\%)$ & 73.7 & $43.3-91.8$ & -0.44 & 0.11 \\
\hline$V_{10}(\%)$ & 67.3 & $34.7-82.5$ & -0.56 & $0.03^{*}$ \\
\hline$V_{15}(\%)$ & 62.5 & $28.8-67.9$ & -0.68 & $<.01^{*}$ \\
\hline$V_{20}(\%)$ & 53.5 & $24.1-64.6$ & -0.76 & $<.01^{*}$ \\
\hline$V_{25}(\%)$ & 46.8 & $20.8-61.1$ & -0.77 & $<.01^{*}$ \\
\hline$V_{30}(\%)$ & 41.9 & $18.3-55.4$ & -0.71 & $<.01^{*}$ \\
\hline$V_{40}(\%)$ & 33.8 & $14.5-47.5$ & -0.65 & $0.01^{*}$ \\
\hline$V_{50}(\%)$ & 20.7 & $6.3-42.1$ & -0.43 & 0.12 \\
\hline$V_{60}(\%)$ & 13.6 & $0-35.5$ & -0.19 & 0.49 \\
\hline Mean irradiated kidney dose (Gy) & 27.5 & $12.9-37.6$ & -0.66 & $<.01^{*}$ \\
\hline Pre-SBRT irradiated renal volume (cc) & 160.4 & $99.4-295.5$ & 0.07 & 0.79 \\
\hline GTV (cc) & 14.8 & $3.0-55.6$ & 0.39 & 0.16 \\
\hline PTV (cc) & 79.8 & $28.1-146.9$ & -0.03 & 0.91 \\
\hline Magnitude of overlap between PTV and kidney volume (cc) & 19.8 & 7.9-50.3 & -0.56 & $0.03^{*}$ \\
\hline post-treatment contralateral kidney volume in percent $(\%, n=12)$ & 99.3 & $89.4-109.0$ & 0.65 & $0.02^{*}$ \\
\hline Isocenter dose (Gy) & 68.8 & $50.0-76.4$ & 0.07 & 0.80 \\
\hline Age (years) & 75 & $58-87$ & 0.03 & 0.89 \\
\hline Creatinine change ratio (peak value/pre-SBRT) (\%) & 139.5 & $105.7-190.0$ & 0.32 & 0.26 \\
\hline
\end{tabular}

Abbreviations: $V_{n}$ percentage volume of the irradiated kidney receiving at least $\mathrm{n}$ dose in $\mathrm{Gy},{ }^{*} p<.05$

Contralateral kidneys were almost unexposed to radiation, and renal atrophy was not seen. Spearman's correlation and Mann-Whitney test were also applied, but no significant factor for post-treatment contralateral kidney volume in percent emerged (data not shown).

\section{Discussion}

This study was one of the few studies in which kidney DVH parameters and other factors were analysed to find predictors of renal atrophy after SBRT and to identify the correlation between post-treatment irradiated kidney volume in percent and kidney $\mathrm{V}_{\mathrm{n}}$. The results showed that $\mathrm{V}_{20}-\mathrm{V}_{30}$ had strong correlation $(r<-0.70, p<.01)$ and that $V_{24}$ had the strongest correlation with renal atrophic change, and a linear equation of estimated post-treatment irradiated kidney volume in percent with
$\mathrm{V}_{24}$ was obtained (Fig. 4). $\mathrm{V}_{10}-\mathrm{V}_{40}$, mean irradiated kidney dose, magnitude of overlap between PTV and kidney volume, and post-treatment contralateral kidney volume in percent had significant relationships with renal atrophy. Patients with implanted fiducial markers showed a significantly lower ratio of renal atrophic change. Compensatory hypertrophy of the contralateral kidney was not observed.

The correlation between $V_{20}$ to $V_{30}$ and renal atrophy was compatible with previous findings, but the values were slightly higher than previous findings considering the number of fractionations [15]. There were some differences from previous reports. First, because SBRT focuses a high dose to a local region with precise respiratory motion management, kidney DVH was less affected by interfractional and intrafractional kidney movement than that

Table 4 Mann-Whitney test between post-treatment irradiated kidney volume in percent and categorical variables

\begin{tabular}{|c|c|c|c|c|c|}
\hline \multirow[b]{2}{*}{ Variables } & \multicolumn{2}{|c|}{ Change ratio of subgroup 1} & \multicolumn{2}{|c|}{ Change ratio of subgroup 2} & \multirow{2}{*}{$\begin{array}{l}\text { Difference } \\
P \text { value }\end{array}$} \\
\hline & Median & Range & Median & Range & \\
\hline Gender (male, female) & 82.8 & $69.4-96.4$ & 75.6 & $613-87.5$ & 0.31 \\
\hline Diabetes mellitus (Yes, No) & 83.4 & $61.3-96.4$ & 82.1 & $69.4-89.5$ & 0.65 \\
\hline Antithrombotic drug (Yes, No) & 82.5 & $69.4-96.4$ & 81.6 & $61.3-95.0$ & 0.88 \\
\hline Fiducial markers (Yes, No) & 87.5 & $75.6-96.4$ & 71.4 & $61.3-83.4$ & $0.02^{*}$ \\
\hline Abdominal compression & 81.7 & $69.4-96.4$ & 82.5 & $61.3-95.0$ & 0.74 \\
\hline 4-dimensional CT (Yes, No) & 82.2 & $61.3-96.4$ & 82.5 & $71.4-89.5$ & 1.00 \\
\hline
\end{tabular}

${ }^{*} p<.05$ 


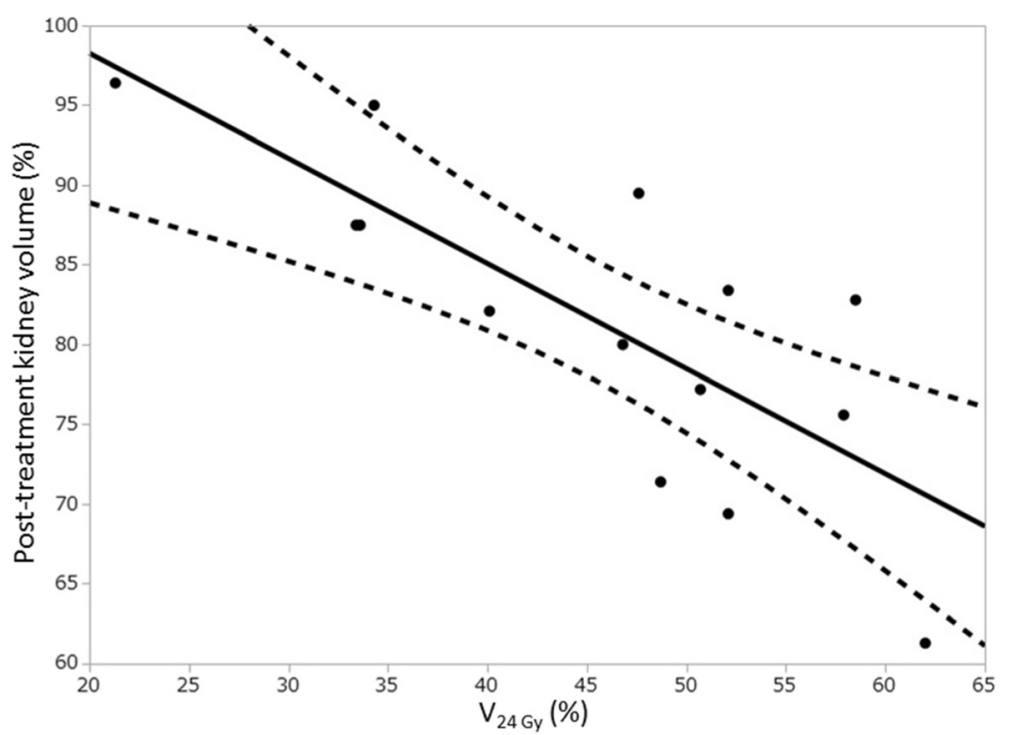

Fig. 4 Scatter plot and estimated linear regression equation. Scatter plot and estimated regression equation as a result of linear regression analysis of posttreatment irradiated kidney volume in percent with $V_{24}$ are shown. Dashed lines represent $95 \%$ confidence bands. The simple linear regression equation is as follows: Estimated post-treatment irradiated kidney volume $(\%)=111.4-0.659 * V_{24}(\%)+\varepsilon$. Epsilon is the error term and its standard deviation is 6.43 .

The coefficient of determination was $0.60(p<.01)$

in conventional fractionation series. Owing to the converged dose distribution, the area outside the PTV and the contralateral kidney were minimally irradiated. Second, no concurrent chemotherapy or adjuvant chemotherapy was used in this study. Third, there were some differences in morphological assessment, such as the measurement of craniocaudal length on CT images [15]. Finally, the analysis in this study was not analysis of bilateral kidneys but analysis of each kidney, and compensatory hypertrophy of the contralateral kidney therefore had less effect on the analysis (but compensatory hypertrophy was not seen in this study) [19]. For either reason, attention must be given to $V_{20}$ to $V_{30}$ of the kidney in a 10 -session scheme of SBRT, but, unfortunately, a clinically meaningful cut-off value was vague in this study because of the limited sample size and various comorbidities.

Although the post-treatment irradiated kidney volume in percent was not correlated with serum creatinine, some surgical series for solitary kidney patients have shown a relationship between renal function and residual kidney volume or volume in percent [18, 20, 21]. Sharma et al. reported that the correlation between percent change in kidney parenchymal volume and percent change in GFR (pre-/postoperative GFR) was moderate but statistically significant. In the present study, only two patients with a solitary kidney were enrolled, and further analysis could therefore not be performed. In a unilateral kidney situation, spared kidney volume from $\mathrm{V}_{\mathrm{n} \text { Gy }}$ may also be important considering surgical results and radiation pneumonitis analyses $[18,22]$.
The post-treatment irradiated kidney volume in percent also had a moderate positive correlation with the posttreatment contralateral kidney volume in percent though this factor was not predicting factor but observed one $(r=$ $0.65, p=0.02$ ). The post-treatment contralateral kidney volume in percent was $99.3 \%$, resulting in no occurrence of the compensatory hypertrophy of contralateral kidney, but that ratio ranged from 89.4 to $109.0 \%$ and had a significant correlation with the post-treatment irradiated kidney volume in percent. This result, that is, a tendency for enlargement of the contralateral kidney with lower posttreatment irradiated kidney volume in percent, suggested that host factors could affect renal remodeling even in a setting with no additional treatment. Patients with little reserved kidney capacity would show more atrophic change because of the irradiation and host factors, and the converse might also occur. Host factors in this study included age and comorbidities such as cardiovascular disease (all of the patients in this study needed administration of an antihypertensive agent and $71 \%$ of the patients needed administration of antithrombotic).

A lower ratio of post-treatment kidney volume was seen in patients with implanted fiducial markers $(p=0.02)$, though prescribed doses were not different $(p=0.54)$. These results were thought to be partly caused by the contribution of fiducial markers to reduction of the internal and set-up margins. In patients with fiducial markers and those without fiducial markers, the mean values of PTV were 84.0 and $83.8 \mathrm{cc}$, respectively $(p=0.99)$, and the mean values of internal volume and set-up volume (i.e., PTV minus GTV) 
were 64.0 and $72.8 \mathrm{cc}$, respectively $(p=0.59)$. In case of $\mathrm{RCC}$, it is often difficult to distinguish the tumor from kidney parenchyma on non-enhanced CT images and even more difficult on non-enhanced cone beam CT images. On the other hand, fiducial markers were easy to identify on non-enhanced cone beam CT images, and the markers allowed us to confirm interfractional and intrafractional motions by using continuous $\mathrm{X}$-ray images of a linear accelerator (on-board imager; Varian Medical Systems, Palo Alto, CA). These facts have probably made radiation oncologists and medical physicists extend the internal and set-up margins when gold fiducial markers were not implanted. Although PTV did not have a relationship with posttreatment irradiated kidney volume in percent, the magnitude of overlap between PTV and kidney volume had a moderate but significant relationship (Table 4). This indicated that not only the internal margin but also location of the tumor in the kidney were important factors for renal atrophy. When the magnitude of overlap between PTV and kidney volume is expected to increase because of the tumor location, implantation of fiducial markers is recommended to reduce the internal margin and renal atrophy.

There are several limitations in the current study. This study was a study conducted in a single institute with a limited sample size, and patient comorbidities were not controlled. Therefore, the exact cut-off value was vague and a clinically meaningful cut-off value was not obtained. The radiotherapy planning CT methods varied, and this affected volumetric analyses to some extent. Studies with a larger sample size and further analyses including multivariate analyses were needed. The periods of assessment CT ranged from 12.0 months to 21.8 months after SBRT. A smaller distribution of SBRT and assessment CT intervals would enable more precise renal atrophy analysis.

\section{Conclusions}

$\mathrm{V}_{10}$ to $\mathrm{V}_{40}$ of the irradiated kidney and mean irradiated kidney dose had significant correlations with the posttreatment irradiated kidney volume in percent, with $\mathrm{V}_{20}$ to $\mathrm{V}_{30}$ showing strong correlations after SBRT for primary RCC. Attention must be paid to the dose distribution at 20 Gy to 30 Gy in 10 fractions in SBRT for RCC. Implantation of fiducial markers might be beneficial to reduce renal atrophy by reducing the internal margin.

\section{Abbreviations}

AAA: analytical anisotropic algorithm; CTV: clinical target volume; DVH: dosevolume histogram; GTV: gross tumor volume; ITV: internal target volume; PTV: planning target volume; RCC: renal cell carcinoma; SBRT: stereotactic body radiotherapy.
}

\section{Acknowledgements}

The authors acknowledge the contributions of doctors in the Department of Urology and doctors in the Department of Radiology in Tohoku University Hospital. They implanted fiducial markers and interpretated CT, MRI and ultrasonic echo findings.
Funding

None.

\section{Authors' contributions}

TY designed the analysis, reviewed the clinical data, performed statistical analysis and drafted the manuscript. NK reviewed the clinical data and gave advice on the manuscript. KT, HM, RU, KS, MK, KI, YI, MK, NT and YK treated the patients, reviewed the clinical data. $\mathrm{HO}$ reviewed the clinical data and gave advice on the manuscript. $\mathrm{KJ}$ treated petients and revised the manuscript. All authors read and approved the final manuscript.

\section{Competing interests}

The authors declare that they have no competing interests.

\section{Consent for publication}

Not applicable.

\section{Ethics approval and consent}

This study was approved by the Ethical Committee of Tohoku University Hospital (reference number: 2011-100), and informed consent was obtained from all patients.

\section{Author details}

${ }^{1}$ Department of Radiation Oncology, Graduate School of Medicine, University of Tohoku, Sendai, Japan. ${ }^{2}$ Radiation Technology, Tohoku University Hospital, Sendai, Japan. ${ }^{3}$ Department of Radiology, School of Medicine, University of Yamanashi, Yamanashi, Japan.

Received: 27 December 2015 Accepted: 17 May 2016

Published online: 26 May 2016

\section{References}

1. Lax I, Blomgren H, Näslund I, Svanström R. Stereotactic radiotherapy of malignancies in the abdomen. Methodological aspects. Acta Oncol. 1994;33:677-83

2. Timmerman R, Paulus R, Galvin J, Michalski J, Straube W, Bradley J, et al. Stereotactic body radiation therapy for inoperable early stage lung cancer. JAMA. 2010;303:1070-6.4

3. Chang JY, Senan S, Paul MA, Mehran RJ, Louie AV, Balter P, et al. Stereotactic ablative radiotherapy versus lobectomy for operable stage I non-small-cell lung cancer: a pooled analysis of two randomised trials. Lancet Oncol. 2015;16:630-7.

4. King CR, Freeman D, Kaplan I, Fuller D, Bolzicco G, Collins S, et al. Stereotactic body radiotherapy for localized prostate cancer: pooled analysis from a multi-institutional consortium of prospective phase II trials. Radiother Oncol. 2013;109:217-21.

5. Herfarth KK, Debus J, Lohr F, Bahner ML, Rhein B, Fritz P, et al. Stereotactic single-dose radiation therapy of liver tumors: results of a phase $\mathrm{I} / \mathrm{II}$ trial. J Clin Oncol. 2001;19:164-70.

6. Hoyer M, Roed H, Sengelov L, Traberg A, Ohlhuis L, Pedersen J, et al. Phase-II study on stereotactic radiotherapy of locally advanced pancreatic carcinoma. Radiother Oncol. 2005;76:48-53.

7. Dawson LA, Kavanagh BD, Paulino AC, Das SK, Miften M, Li XA, et al. Radiation-associated kidney injury. Int J Radiat Oncol Biol Phys. 2010;76(3 Suppl):S108-15

8. NCCN. Clinical practice guidelines in oncology [2015 ver 3]. Available from: http://www.nccn.org/professionals/physician_gls/pdf/kidney.pdf. Accessed 28 Dec 2015

9. Tunio MA, Hashmi A, Rafi M. Need for a new trial to evaluate postoperative radiotherapy in renal cell carcinoma: a meta-analysis of randomized controlled trials. Ann Oncol. 2010;21:1839-45.

10. Siva S, Pham D, Gill S, Corcoran NM, Foroudi F. A systematic review of stereotactic radiotherapy ablation for primary renal cell carcinoma. BJU Int. 2012;110:E737-43.

11. Pham D, Thompson A, Kron T, Foroudi F, Kolsky MS, Devereux T, et al. Stereotactic ablative body radiation therapy for primary kidney cancer: a 3-dimensional conformal technique associated with low rates of early toxicity. Int J Radiat Oncol Biol Phys. 2014;90:1061-8.

12. Staehler M, Bader M, Schlenker B, Casuscelli J, Karl A, Roosen A, et al. Single fraction radiosurgery for the treatment of renal tumors. J Urol. 2015;193:771-5. 
13. Ponsky L, Lo SS, Zhang Y, Schluchter M, Liu Y, Patel R, et al. Phase I dose-escalation study of stereotactic body radiotherapy (SBRT) for poor surgical candidates with localized renal cell carcinoma. Radiother Oncol. 2015:117:183-7.

14. Phase I/II study of stereotactic radiotherapy for renal cell cancer. Available from: https://upload.umin.ac.jp/cgi-open-bin/ctr/ctr.cgi?function=brows \&action $=$ brows\&recptno $=R 000005008 \&$ type $=$ summary\&language $=E$. Accessed 28 Dec 2015.

15. Yang GY, May KS, lyer RV, Chandrasekhar R, Wilding GE, McCloskey SA, et al. Renal atrophy secondary to chemoradiotherapy of abdominal malignancies. Int J Radiat Oncol Biol Phys. 2010;78:539-46.

16. Jackson P, Foroudi F, Pham D, Hofman MS, Hardcastle N, Callahan J, et al. Short communication: timeline of radiation-induced kidney function loss after stereotactic ablative body radiotherapy of renal cell carcinoma as evaluated by serial (99 m)Tc-DMSA SPECT/CT. Radiat Oncol. 2014;9:253.

17. Svedman C, Karlsson K, Rutkowska E, Sandström P, Blomgren H, Lax I, et al. Stereotactic body radiotherapy of primary and metastatic renal lesions for patients with only one functioning kidney. Acta Oncol. 2008;47:1578-83.

18. Kuru TH, Zhu J, Popeneciu IV, Rudhardt NS, Hadaschik BA, Teber D, et al. Volumetry may predict early renal function after nephron sparing surgery in solitary kidney patients. Springerplus. 2014;3:488.

19. Dewit L, Verheij M, V Olmos RA, Arisz L. Compensatory renal response after unilateral partial and whole volume high-dose irradiation of the human kidney. Eur J Cancer Part A Gen Top. 1993:29:2239-43.

20. Sharma N, O'Hara J, Novick AC, Lieber M, Remer EM, Herts BR. Correlation between loss of renal function and loss of renal volume after partial nephrectomy for tumor in a solitary kidney. J Urol. 2008;179:1284-8.

21. Thompson RH, Lane BR, Lohse CM, Leibovich BC, Fergany A, Frank I, et al. Renal function after partial nephrectomy: effect of warm ischemia relative to quantity and quality of preserved kidney. Urology. 2012;79:356-60.

22. Tsujino K, Hashimoto T, Shimada T, Yoden E, Fujii O, Ota Y, et al. Combined analysis of V20, VS5, pulmonary fibrosis score on baseline computed tomography, and patient age improves prediction of severe radiation pneumonitis after concurrent chemoradiotherapy for locally advanced non-small-cell lung cancer. J Thorac Oncol. 2014;9:983-90.

\section{Submit your next manuscript to BioMed Central and we will help you at every step:}

- We accept pre-submission inquiries

- Our selector tool helps you to find the most relevant journal

- We provide round the clock customer support

- Convenient online submission

- Thorough peer review

- Inclusion in PubMed and all major indexing services

- Maximum visibility for your research

Submit your manuscript at www.biomedcentral.com/submit

C Biomed Central 